\title{
AN EXPONENTIAL CONTINUOUS-TIME GARCH PROCESS
}

\author{
STEPHAN HAUG ${ }^{* * *}$ AND \\ CLAUDIA CZADO, ${ }^{* * * *}$ Munich University of Technology
}

\begin{abstract}
In this paper we introduce an exponential continuous-time $\operatorname{GARCH}(p, q)$ process. It is defined in such a way that it is a continuous-time extension of the discrete-time $\operatorname{EGARCH}(p, q)$ process. We investigate stationarity, mixing, and moment properties of the new model. An instantaneous leverage effect can be shown for the exponential continuous-time $\operatorname{GARCH}(p, p)$ model.
\end{abstract}

Keywords: Exponential continuous-time GARCH process; EGARCH; Lévy process; stationarity; leverage effect; stochastic volatility

2000 Mathematics Subject Classification: Primary 60G10; 60G12; 91B70

Secondary 91B 84

\section{Introduction}

GARCH-type processes have become very popular in financial econometrics to model returns of stocks, exchange rates, and other series observed at equidistant time points. They have been designed (see [9] and [3]) to capture so-called stylised facts of such data, which are, for example, volatility clustering, dependence without correlation, and tail heaviness. Another characteristic is that stock returns seem to be negatively correlated with changes in the volatility, i.e. that volatility tends to increase after negative shocks and to decrease after positive shocks. This effect is called the leverage effect and cannot be modelled by a GARCH-type process without further extensions. This finding led Nelson [19] to introduce the exponential GARCH process, which is able to model this asymmetry in stock returns. The log-volatility of the $\operatorname{EGARCH}(p, q)$ process was modelled as an $\operatorname{ARMA}(q, p-1)$ process. Another popular model in this context is the linear ARCH process suggested by Robinson [21]. Here the volatility process is modelled as the square of an $\mathrm{MA}(\infty)$ process driven by past observations. In [10] it was shown that the model contains, in addition to a long memory property, a leverage effect.

The availability of high-frequency data, which has increased enormously in the last few years, is one reason for considering continuous-time models with similar behaviour to discretetime GARCH models. The reason for this is that at the highest available frequency, the observations of the price process occur at irregularly spaced time points and, therefore, it is natural to assume an underlying continuous-time model. Different approaches have been taken to set up a continuous-time model, which has the same features as discrete-time GARCH processes. Recently Klüppelberg et al. [13] developed a continuous-time GARCH(1, 1) model, abbreviated to a COGARCH $(1,1)$ model in the sequel. Their approach fundamentally differed from previous attempts, which could be summarised as diffusion approximations (see, e.g. [18]),

Received 13 September 2006; revision received 13 September 2007.

* Postal address: Center for Mathematical Sciences, Munich University of Technology, D-85747 Garching, Germany.

** Email address: haug@ma.tum.de

*** Email address: cczado@ma.tum.de 
by the fact that their model was driven by only one source of randomness (as in discrete-time GARCH models) instead of two (as in the diffusion approximations). They replaced the noise process of discrete-time GARCH models by the jumps of a Lévy process. The $\operatorname{COGARCH}(1,1)$ model was then extended by Brockwell et al. [6] to a continuous-time $\operatorname{GARCH}(p, q)$ process for general orders $p, q \in \mathbb{N}, q \geq p$; henceforth called a $\operatorname{COGARCH}(p, q)$ process.

In this paper a continuous-time analogue of the $\operatorname{EGARCH}(p, q)$ model is introduced. The noise processes will also be modelled by the increments of a Lévy process. As in the discretetime case, we describe the log-volatility process as a linear process, more precisely a continuoustime $\operatorname{ARMA}(q, p-1)$ process.

The paper is organised as follows. In Section 2 we review the definition of the discrete-time EGARCH process. After a short review of the elementary properties of Lévy processes, we define the exponential continuous-time $\operatorname{GARCH}(p, q)$ process at the beginning of Section 3 . In addition, we state stationarity conditions for the log-volatility and volatility processes of our model. Afterwards, the leverage effect in our model is considered. We close Section 3 with an investigation of the mixing properties of the (log-)volatility and return processes. In Section 4 we derive second-order properties of the volatility process. Section 5 is devoted to the analysis of the second-order behaviour of the return process. We derive expressions for the first and second moment of the return process. The stylised fact of zero correlation in the return process, but correlation of the squared returns, is also shown.

\section{The discrete-time EGARCH process}

Motivated by empirical evidence that stock returns are negatively correlated with changes in returns volatility, Nelson [19] defined the exponential GARCH process (EGARCH) to model this effect, which is called the leverage effect (see also Section 3.1).

Definition 2.1. The process $\left(X_{n}\right)_{n \in \mathbb{Z}}$ of the form $X_{n}=\sigma_{n} \varepsilon_{n}, n \in \mathbb{Z}$, where $\left(\varepsilon_{n}\right)_{n \in \mathbb{Z}}$ is an independent and identically distributed (i.i.d.) sequence with $\mathrm{E}\left(\varepsilon_{1}\right)=0$ and $\operatorname{var}\left(\varepsilon_{1}\right)=1$, is called an EGARCH process, if the volatility process $\left(\sigma_{n}^{2}\right)_{n \in \mathbb{Z}}$ satisfies

$$
\log \left(\sigma_{n}^{2}\right)=\mu+\sum_{k=1}^{\infty} \beta_{k} f\left(\varepsilon_{n-k}\right)
$$

where $f: \mathbb{R} \rightarrow \mathbb{R}$ is some measurable real-valued deterministic function, $\mu \in \mathbb{R}$, and $\left(\beta_{k}\right)_{k \in \mathbb{N}}$ are real coefficients such that $\mathrm{E}\left(\left|f\left(\varepsilon_{n}\right)\right|\right)<\infty, \operatorname{var}\left(f\left(\varepsilon_{n}\right)\right)<\infty$, and $\sum_{k=1}^{\infty}\left|\beta_{k}\right|<\infty$.

Nelson [19] also suggested a finite-parameter model by modelling the log-volatility as an $\operatorname{ARMA}(q, p-1)$ process instead of an infinite-moving average process. This leads to the $\operatorname{EGARCH}(p, q)$ model, which is defined in the following way.

Definition 2.2. Let $p, q \in \mathbb{N}$ and $\mu, \alpha_{1}, \ldots, \alpha_{q}, \beta_{1}, \ldots, \beta_{p} \in \mathbb{R}$. Suppose that $\alpha_{q} \neq 0$, $\beta_{p} \neq 0$, that the autoregressive polynomial $\phi(z):=1-\alpha_{1} z-\cdots-\alpha_{q} z^{q}$ and the moving average polynomial $\psi(z):=\beta_{1}+\beta_{2} z+\cdots+\beta_{p} z^{p-1}$ have no common zeros, and that $\phi(z) \neq 0$ on $\{z \in \mathbb{C}|| z \mid \leq 1\}$. Let $\left(\varepsilon_{n}\right)_{n \in \mathbb{Z}}$ be an i.i.d. sequence with $\mathrm{E}\left(\varepsilon_{1}\right)=0$ and $\operatorname{var}\left(\varepsilon_{1}\right)=1$, and let $f(\cdot)$ be such that $\mathrm{E}\left(\left|f\left(\varepsilon_{n}\right)\right|\right)<\infty$ and $\operatorname{var}\left(f\left(\varepsilon_{n}\right)\right)<\infty$. Then $\left(X_{n}\right)_{n \in \mathbb{Z}}$, where $X_{n}=\sigma_{n} \varepsilon_{n}$ and

$$
\log \left(\sigma_{n}^{2}\right)=\mu+\sum_{k=1}^{p} \beta_{k} f\left(\varepsilon_{n-k}\right)+\sum_{k=1}^{q} \alpha_{k} \log \left(\sigma_{n-k}^{2}\right),
$$

is called an $\operatorname{EGARCH}(p, q)$ process. 
To achieve the asymmetric relation between the stock returns and the volatility, $f\left(\varepsilon_{n}\right)$ must be a function of the magnitude and the sign of $\varepsilon_{n}$, as noted by Nelson [19]. Therefore, he proposed the following function:

$$
f\left(\varepsilon_{n}\right):=\theta \varepsilon_{n}+\gamma\left(\left|\varepsilon_{n}\right|-\mathrm{E}\left(\left|\varepsilon_{n}\right|\right)\right),
$$

with real coefficients $\theta$ and $\gamma$. We see that $f\left(\varepsilon_{n}\right)$ is piecewise linear in $\varepsilon_{n}$ and has slope $\theta+\gamma$ for positive shocks $\varepsilon_{n}$ and slope $\theta-\gamma$ for negative shocks. Therefore, $f\left(\varepsilon_{n}\right)$ allows the volatility process $\left(\sigma_{n}^{2}\right)_{n \in \mathbb{Z}}$ to respond asymmetrically to positive and negative jumps in the stock price.

\section{The exponential COGARCH process}

The goal of this section is to construct a continuous-time analogue of the discrete-time $\operatorname{EGARCH}(p, q)$ process. Therefore, we will use the idea of Klüppelberg et al. [13] to replace the noise variables $\varepsilon_{n}$ by the increments of a Lévy process $L=\left(L_{t}\right)_{t \geq 0}$. Any Lévy process $L$ on $\mathbb{R}$ has a characteristic function of the form $\mathrm{E}\left(\exp \left(\mathrm{i} u L_{t}\right)\right)=\exp \left\{t \psi_{L}(u)\right\}, t \geq 0$, with

$$
\psi_{L}(u):=\mathrm{i} \gamma_{L} u-\frac{\tau_{L}^{2}}{2} u^{2}+\int_{\mathbb{R}}\left(\mathrm{e}^{\mathrm{i} u x}-1-\mathrm{i} u x \chi_{(-1,1)}(x)\right) \nu_{L}(\mathrm{~d} x), \quad u \in \mathbb{R},
$$

where $\tau_{L}^{2} \geq 0, \gamma_{L} \in \mathbb{R}$, the measure $\nu_{L}$ satisfies

$$
v_{L}(\{0\})=0 \text { and } \int_{\mathbb{R}} \min \left(x^{2}, 1\right) v_{L}(\mathrm{~d} x)<\infty,
$$

and $\chi_{A}(\cdot)$ denotes the indicator function of the set $A \subset \mathbb{R}$. The measure $v_{L}$ is called the Lévy measure of $L$, and the triplet $\left(\gamma_{L}, \tau_{L}^{2}, v_{L}\right)$ is called the characteristic triplet of $L$. The map $\psi_{L}$ is called the Lévy symbol . For more details on Lévy processes, we refer the reader to [1] or [22].

We consider Lévy processes $L$ defined on a probability space $(\Omega, \mathcal{F}, \mathrm{P})$ with jumps $\Delta L_{t}:=$ $L_{t}-L_{t-}$, zero mean, and finite variance. In this case the Lévy-Itô decomposition (see, e.g. [1, Theorem 2.4.16]) of $L$ is

$$
L_{t}=B_{t}+\int_{\mathbb{R} \backslash\{0\}} x \tilde{N}_{L}(t, \mathrm{~d} x), \quad t \geq 0,
$$

where $B$ is a Brownian motion with variance $\tau_{L}^{2}$, and $\tilde{N}_{L}(t, \mathrm{~d} x)=N_{L}(t, \mathrm{~d} x)-t v_{L}(\mathrm{~d} x), t \geq 0$, is the compensated random measure associated to the Poisson random measure

$$
N_{L}(t, A)=\#\left\{0 \leq s<t ; \Delta L_{s} \in A\right\}=\sum_{0<s \leq t} \chi_{A}\left(\Delta L_{s}\right), \quad A \in \mathscr{B}(\mathbb{R} \backslash\{0\}),
$$

on $\mathbb{R}_{+} \times \mathbb{R} \backslash\{0\}$, which is independent of $B$.

The driving-noise process of the log-volatility in this continuous-time model will be constructed similarly to the discrete-time case. In particular, for a zero-mean Lévy process $L$, with $\mathrm{E}\left(L_{1}^{2}\right)<\infty$ and parameters $(\theta, \gamma)^{\top} \in \mathbb{R}^{2} \backslash\{\boldsymbol{0}\}$, we define the driving process $M$ of the log-volatility process by

$$
M_{t}:=\int_{\mathbb{R} \backslash\{0\}} h(x) \tilde{N}_{L}(t, \mathrm{~d} x), \quad t \geq 0,
$$

with $h(x):=\theta x+\gamma|x|$. 
Remark 3.1. (i) The process $M$ defined by (3.1) is by construction a process with independent and stationary increments and by Theorem 4.3 .4 of [1] well defined if

$$
\int_{\mathbb{R}}|h(x)|^{2} v_{L}(\mathrm{~d} x)<\infty .
$$

Condition (3.2) is satisfied since $v_{L}$ is a Lévy measure and $L$ has finite variance. By Equation (2.9) of [1], the characteristic triplet of $M$ is $\left(\gamma_{M}, 0, v_{M}\right)$, where $v_{M}:=v_{L} \circ h^{-1}$ is the Lévy measure of $M$ and $\gamma_{M}:=-\int_{|x|>1} x v_{M}(\mathrm{~d} x)$. The precise form of $v_{M}$ depends on the sign and size of $\theta$ and $\gamma$, and is given in the following formulae:

$$
v_{M}((-\infty,-x])= \begin{cases}v_{L}\left(\left[-\frac{x}{\theta+\gamma}, \infty\right)\right)+v_{L}\left(\left(-\infty,-\frac{x}{\theta-\gamma}\right]\right), & -\gamma>\theta>\gamma, \\ v_{L}\left(\left(-\infty,-\frac{x}{\theta-\gamma}\right]\right), & -\theta<\gamma<\theta, \\ v_{L}\left(\left[-\frac{x}{\theta+\gamma}, \infty\right)\right), & -\theta>\gamma>\theta, \\ 0, & -\gamma<\theta<\gamma,\end{cases}
$$

and

$$
v_{M}([x, \infty))= \begin{cases}v_{L}\left(\left[\frac{x}{\theta+\gamma}, \infty\right)\right)+v_{L}\left(\left(-\infty, \frac{x}{\theta-\gamma}\right]\right), & -\gamma<\theta<\gamma, \\ v_{L}\left(\left(-\infty, \frac{x}{\theta-\gamma}\right]\right), & -\theta>\gamma>\theta, \\ v_{L}\left(\left[\frac{x}{\theta+\gamma}, \infty\right)\right), & -\theta<\gamma<\theta, \\ 0, & -\gamma>\theta>\gamma,\end{cases}
$$

for $x>0$. We recognise that $M$ is a spectrally negative Lévy process for $\gamma<\theta<-\gamma$, i.e. $M$ has only negative jumps, and a spectrally positive Lévy process for $-\gamma<\theta<\gamma$.

(ii) In case the jump part of $L$ is of finite variation, $M$ is a Lévy process of finite variation with Lévy-Itô decomposition

$$
M_{t}:=\sum_{0<s \leq t}\left[\theta \Delta L_{s}+\gamma\left|\Delta L_{s}\right|\right]-C t, \quad t>0,
$$

where $C:=\gamma \int_{\mathbb{R}}|x| \nu_{L}(\mathrm{~d} x)$.

Now we define the exponential continuous-time $\operatorname{GARCH}(p, q)$ process by specifying the $\log$-volatility process as a continuous-time $\operatorname{ARMA}(q, p-1)$ process; henceforth called a $\operatorname{CARMA}(q, p-1)$ process (see, e.g. [5] for details on CARMA processes), which is the continuous-time analogue of an $\operatorname{ARMA}(q, p-1)$ process.

Definition 3.1. Let $L=\left(L_{t}\right)_{t \geq 0}$ be a zero-mean Lévy process with Lévy measure $v_{L}$ such that $\int_{|x| \geq 1} x^{2} v_{L}(\mathrm{~d} x)<\infty$. Then we define the exponential $\operatorname{COGARCH}(p, q)$ process $G$, abbreviated to $\operatorname{ECOGARCH}(p, q)$, as the stochastic process satisfying

$$
\mathrm{d} G_{t}:=\sigma_{t-} \mathrm{d} L_{t}, \quad t>0, G_{0}=0,
$$


where the $\log$-volatility process $\log \left(\sigma^{2}\right)=\left(\log \left(\sigma_{t}^{2}\right)\right)_{t \geq 0}$ is a $\operatorname{CARMA}(q, p-1)$ process, $1 \leq p \leq q$, with mean $\mu \in \mathbb{R}$ and state space representation

$$
\begin{gathered}
\log \left(\sigma_{t}^{2}\right):=\mu+\boldsymbol{b}^{\top} \boldsymbol{X}_{t}, \quad t \geq 0, \\
\mathrm{~d} \boldsymbol{X}_{t}=\boldsymbol{A} \boldsymbol{X}_{t} \mathrm{~d} t+\mathbf{1}_{q} \mathrm{~d} M_{t}, \quad t>0,
\end{gathered}
$$

where $\boldsymbol{X}_{0} \in \mathbb{R}^{q}$ is independent of the driving Lévy process $M$. The $q \times q$ matrix $\boldsymbol{A}$ and the vectors $\boldsymbol{b} \in \mathbb{R}^{q}$ and $\mathbf{1}_{q} \in \mathbb{R}^{q}$ are defined by

$$
\boldsymbol{A}=\left[\begin{array}{ccccc}
0 & 1 & 0 & \cdots & 0 \\
0 & 0 & 1 & \cdots & 0 \\
\vdots & \vdots & \vdots & \ddots & \vdots \\
0 & 0 & 0 & \cdots & 1 \\
-a_{q} & -a_{q-1} & -a_{q-2} & \cdots & -a_{1}
\end{array}\right], \quad \boldsymbol{b}=\left[\begin{array}{c}
b_{1} \\
b_{2} \\
\vdots \\
b_{q-1} \\
b_{q}
\end{array}\right], \quad \mathbf{1}_{q}=\left[\begin{array}{c}
0 \\
0 \\
\vdots \\
0 \\
1
\end{array}\right]
$$

with coefficients $a_{1}, \ldots, a_{q}, b_{1}, \ldots, b_{p} \in \mathbb{R}$, where $a_{q} \neq 0, b_{p} \neq 0$, and $b_{p+1}=\cdots=$ $b_{q}=0$.

Returns over a time interval of length $r>0$ are described by the increments of $G$ :

$$
G_{t}^{(r)}:=G_{t}-G_{t-r}=\int_{(t-r, t]} \sigma_{s-} \mathrm{d} L_{s}, \quad t \geq r>0 .
$$

Thus, this gives us the possibility to model ultra-high-frequency data, which consists of returns over varying time intervals. Conversely, an equidistant sequence of such nonoverlapping returns of length $r$ is given by $\left(G_{n r}^{(r)}\right)_{n \in \mathbb{N}}$.

In the sequel we refer to $G$ and $G^{(r)}$ as the (log-)price process and the (log-)return process, respectively. Also, we call $\sigma^{2}$ and $\log \left(\sigma^{2}\right)$ the volatility process and the log-volatility process, respectively.

Remark 3.2. ([5, Proposition 2].) (i) As the log-volatility process is a $\operatorname{CARMA}(q, p-1)$ process, it is strictly stationary if the eigenvalues of $\boldsymbol{A}$ all have negative real parts and $\boldsymbol{X}_{0}$ has the same distribution as $\int_{0}^{\infty} \mathrm{e}^{\boldsymbol{A} u} \mathbf{1}_{q} \mathrm{~d} M_{u}$. If $\sigma^{2}$ is as defined in Definition 3.1 then $\sigma^{2}$ is also strictly stationary, as this property is preserved under measurable mappings.

(ii) The solution of the continuous-time state-space model, (3.3) and (3.4), has the representation

$$
\log \left(\sigma_{t}^{2}\right)=\mu+\boldsymbol{b}^{\top} \mathrm{e}^{\boldsymbol{A} t} \boldsymbol{X}_{0}+\int_{0}^{t} \boldsymbol{b}^{\top} \mathrm{e}^{\boldsymbol{A}(t-u)} \mathbf{1}_{q} \mathrm{~d} M_{u}, \quad t>0 .
$$

If we choose a second Lévy process, $\left(\tilde{L}_{t}\right)_{t \geq 0}$, independent of $L$ and with the same distribution as $L$, then we can define an extension $\left(L_{t}^{*}\right)_{t \in \mathbb{R}}$ of $L$ to the real line by

$$
L_{t}^{*}:=L_{t} \chi_{[0, \infty)}(t)-\tilde{L}_{-t-\chi} \chi_{(-\infty, 0)}(t), \quad t \in \mathbb{R} .
$$

Using $L^{*}$ instead of $L$ in (3.1), we obtain an extension $M^{*}$ of $M$. For simplicity, in the following we will write $L$ and $M$ instead of $L^{*}$ and $M^{*}$. In the strictly stationary case the log-volatility process can be defined on the whole real line by

$$
\log \left(\sigma_{t}^{2}\right)=\mu+\int_{-\infty}^{t} g(t-u) \mathrm{d} M_{u}, \quad t \in \mathbb{R}
$$


with kernel function

$$
g(t)=\boldsymbol{b}^{\top} \mathrm{e}^{A t} \mathbf{1}_{q} \chi_{(0, \infty)}(t)
$$

see $[5$, Section 2] for further details.

(iii) From (3.5) it directly follows that the increments $G^{(r)}=\int_{(\cdot-r, \cdot]} \sigma_{s-} \mathrm{d} L_{s}$ of $G$ are stationary if the volatility $\sigma^{2}$ is stationary, as the increments of $L$ are stationary and independent by definition.

(iv) If $q \geq p+1$, the log-volatility process is $(q-p-1)$ times differentiable, which follows from the state-space representation of $\log \left(\sigma^{2}\right)$ and, hence, the volatility process has continuous sample paths. In particular, the volatility will contain jumps only for $p=q$.

\subsection{The leverage effect}

For empirical return data, researchers have found evidence that (see, e.g. [19, Section 1]) current returns are negatively correlated with future volatility. We distinguish two cases. Either a negative jump in the log-price process $G$ increases the future volatility more than a positive jump of the same size (compare to (i), below, with $-\gamma<\theta<0$ ), or a negative jump in $G$ increases the future volatility while a positive jump even decreases it (compare to (iv), below). This phenomenon is called the leverage effect in the literature.

If we take a look at the jumps of the state process $\boldsymbol{X}$ in the $\operatorname{ECOGARCH}(p, q)$ model,

$$
\Delta M_{t}= \begin{cases}(\theta+\gamma) \Delta L_{t}, & \Delta L_{t} \geq 0, \\ (\theta-\gamma) \Delta L_{t}, & \Delta L_{t}<0,\end{cases}
$$

we see that

(i) for $-\gamma<\theta<0(0<\theta<\gamma)$, a positive jump $\Delta L_{t}$ leads to a smaller (greater) positive jump $\Delta M_{t}$ compared to a negative jump $\Delta L_{t}$ of the same size;

(ii) for $\theta>|\gamma|$, a positive jump $\Delta L_{t}$ leads to a positive jump $\Delta M_{t}$, while a negative jump of the same size results in a negative jump $\Delta M_{t}$;

(iii) for $0<\theta<-\gamma(\gamma<\theta<0)$, a positive jump $\Delta L_{t}$ leads to a smaller (greater) negative jump $\Delta M_{t}$ compared to a negative jump $\Delta L_{t}$ of the same size;

(iv) for $\theta<-|\gamma|$, a positive jump $\Delta L_{t}$ leads to a negative jump $\Delta M_{t}$, while a negative jump of the same size results in a positive jump $\Delta M_{t}$.

If we compare this to the $\operatorname{COGARCH}(p, q)$ process, we see that in the COGARCH model the innovations of the volatility process at time $t$ are given by the squared innovations of the log-price process (see [6, Section 2]). Hence, the volatility process of the COGARCH model reacts in the same way to positive and negative jumps. Now we will consider the instantaneous leverage effect, which is defined as

$$
\operatorname{cov}\left(\Delta G_{t}, \sigma_{t}^{2}|| \Delta L_{t} \mid>\varepsilon\right)
$$

being negative. Intuitively it is clear that this correlation can only be different from 0 if the sample paths of $\sigma^{2}$ exhibit jumps. But, from Remark 3.2(iv) we know that this is the case only for $p=q$. The reason is that, for $p<q$, the parameter $b_{q}$ will be 0 and, therefore, the jump $\Delta L_{t}$ at time $t$ contributes only to the $(q-1)$ th derivative of the state process $\boldsymbol{X}$, but is not taken into account for the log-volatility at that time point. Thus, we will expect an instantaneous 
leverage effect only for the $\operatorname{ECOGARCH}(p, p)$ models. This will be shown in Proposition 3.1, below; in particular, we will show that the sign of the correlation is equal to the sign of $\theta b_{q}$. This result is similar to the discrete-time case; see [23, Proposition 2.9].

Proposition 3.1. Assume that the distribution of the jumps of $L$ is symmetric, i.e. for all $\varepsilon>0$,

$$
\mathrm{P}\left(\Delta L_{t} \in \mathrm{d} x|| \Delta L_{t} \mid>\varepsilon\right)=\mathrm{P}\left(\Delta L_{t} \in-\mathrm{d} x|| \Delta L_{t} \mid>\varepsilon\right), \quad t \geq 0 .
$$

Conditionally on the event that $\left|\Delta L_{t}\right|>\varepsilon$, the sign of $\operatorname{cov}\left(\Delta G_{t}, \sigma_{t}^{2}\right)$ is equal to the sign of $\theta b_{q}$.

Proof. Since the distribution of the jumps of $L$ is symmetric, we obtain

$$
\mathrm{E}\left(\Delta G_{t}|| \Delta L_{t} \mid>\varepsilon\right)=\mathrm{E}\left(\sigma_{t-}\right) \mathrm{E}\left(\Delta L_{t}|| \Delta L_{t} \mid>\varepsilon\right)=0 .
$$

This then implies that

$$
\begin{aligned}
\operatorname{cov}\left(\Delta G_{t}, \sigma_{t}^{2}|| \Delta L_{t} \mid>\varepsilon\right) & =\mathrm{E}\left(\Delta G_{t} \sigma_{t}^{2}|| \Delta L_{t} \mid>\varepsilon\right) \\
& =\mathrm{E}\left(\Delta G_{t} \exp \left(\log \left(\sigma_{t-}^{2}\right)+b_{q} \Delta M_{t}\right)|| \Delta L_{t} \mid>\varepsilon\right) \\
& =\mathrm{E}\left(\sigma_{t-}^{3} \Delta L_{t} \exp \left(b_{q}\left(\theta \Delta L_{t}+\gamma\left|\Delta L_{t}\right|\right)\right)|| \Delta L_{t} \mid>\varepsilon\right) .
\end{aligned}
$$

Since $\Delta L_{t}$ is independent of $\sigma_{t-}^{3}$, we obtain

$$
\begin{aligned}
& \operatorname{cov}\left(\Delta G_{t}, \sigma_{t}^{2}|| \Delta L_{t} \mid>\varepsilon\right) \\
& \quad=\mathrm{E}\left(\sigma_{t-}^{3}\right) \mathrm{E}\left(\Delta L_{t} \exp \left(b_{q}\left(\theta \Delta L_{t}+\gamma\left|\Delta L_{t}\right|\right)\right)|| \Delta L_{t} \mid>\varepsilon\right) \\
& \quad=\mathrm{E}\left(\sigma_{t-}^{3}\right) \int_{x>\varepsilon} x \exp \left(b_{q} \gamma x\right)\left(\exp \left(\theta b_{q} x\right)-\exp \left(-\theta b_{q} x\right)\right) \mathrm{P}\left(\Delta L_{t} \in \mathrm{d} x|| \Delta L_{t} \mid>\varepsilon\right) .
\end{aligned}
$$

From the fact that $\operatorname{sgn}\left(\exp \left(\theta b_{q} x\right)-\exp \left(-\theta b_{q} x\right)\right)=\operatorname{sgn}\left(\theta b_{q}\right)$ for all $x>\varepsilon$, the desired result follows.

From Proposition 3.1 we see that the existence of an instantaneous leverage effect depends only on the parameters $\theta$ and $b_{q}$. Hence, we will have a leverage effect for all orders $p \geq 1$ as long as the condition on $\theta$ and $b_{q}$ is satisfied. In simulations of the $\operatorname{ECOGARCH}(p, p)$ process we see similar results concerning the leverage effect for different orders $p \geq 1$. As an example, we will now consider an $\operatorname{ECOGARCH}(1,1)$ process.

Example 3.1. In this example we consider an $\operatorname{ECOGARCH}(1,1)$ process driven by a Lévy process $L$ with Lévy symbol

$$
\psi_{L}(u)=-\frac{u^{2}}{2}+\int_{\mathbb{R}}\left(\mathrm{e}^{\mathrm{i} u x}-1\right) \lambda \Phi_{0,1 / \lambda}(\mathrm{d} x),
$$

where $\Phi_{0,1 / \lambda}(\cdot)$ is the distribution function of a normal distribution with zero mean and variance $1 / \lambda$. This means that $L$ is the sum of a standard Brownian motion $W$ and the compound Poisson process $J_{t}=\sum_{k=1}^{N_{t}} Z_{k}, t \geq 0$, where $\left(N_{t}\right)_{t \in \mathbb{R}}$ is an independent Poisson process with intensity $\lambda>0$ and jump times $\left(T_{k}\right)_{k \in \mathbb{Z}}$. The Poisson process $N$ is also independent from the i.i.d. sequence of jump sizes $\left(Z_{k}\right)_{k \in \mathbb{Z}}$, with $Z_{1} \sim N(0,1 / \lambda)$. The Lévy process $M$ is, in this case, 

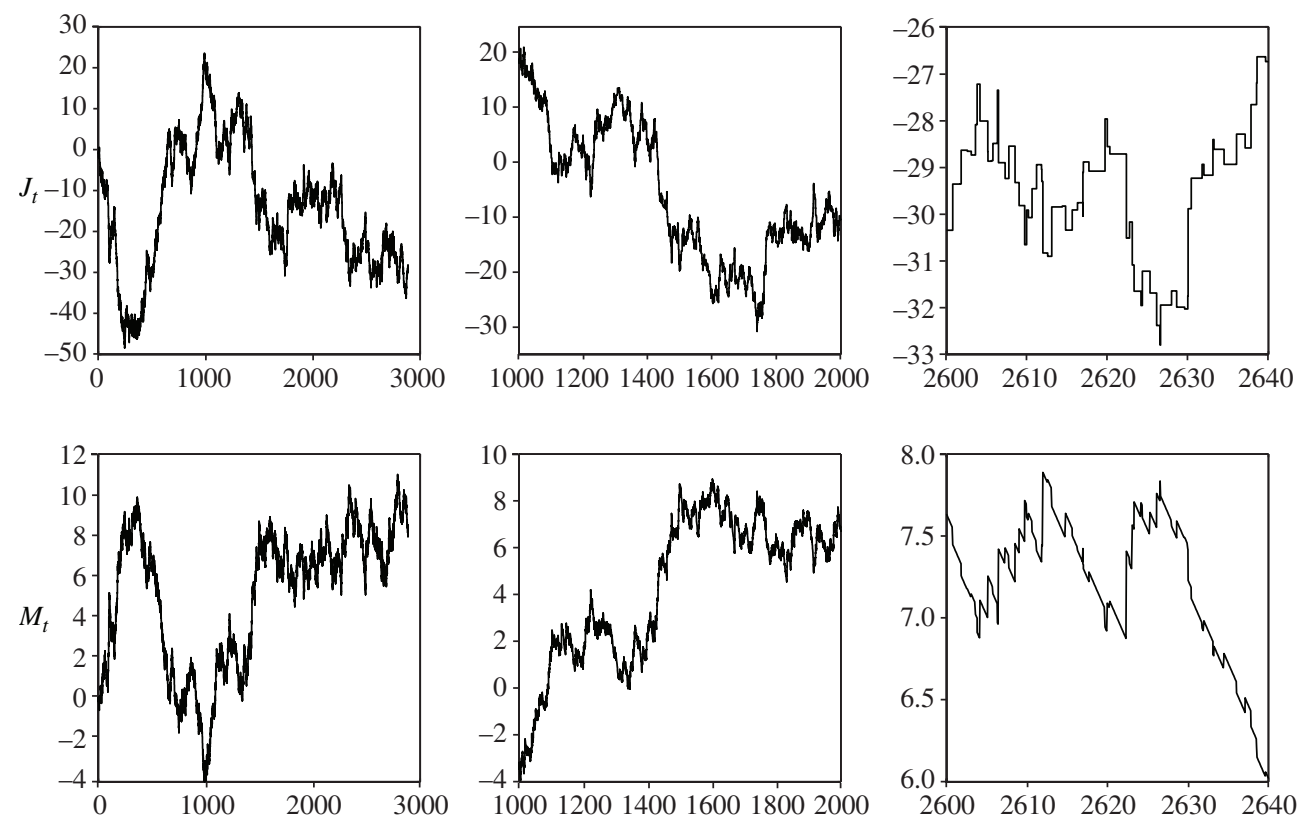

FIGURE 1: Simulated sample paths of $J$ (top row) and $M$ (bottom row), with parameters $\theta=-0.2$ and $\gamma=0.1$, over three different time scales.

given by the following expression:

$$
M_{t}=\sum_{k=1}^{N_{t}}\left[\theta Z_{k}+\gamma\left|Z_{k}\right|\right]-C t, \quad t>0,
$$

with

$$
C=\gamma \int_{\mathbb{R}}|x| \lambda \Phi_{0,1 / \lambda}(\mathrm{d} x)=\gamma \sqrt{\frac{2 \lambda}{\pi}} .
$$

If we just consider the case in which $\theta<-\gamma<0$ then the Lévy measure $v_{M}$ of $M$ is defined by

$$
v_{M}((-\infty,-x])=\lambda \Phi_{0,1 / \lambda}\left(\left[-\frac{x}{\theta+\gamma}, \infty\right)\right), \quad x>0,
$$

on the negative half real line and by

$$
v_{M}([x, \infty))=\lambda \Phi_{0,1 / \lambda}\left(\left(-\infty, \frac{x}{\theta-\gamma}\right]\right), \quad x>0,
$$

on the positive half real line. In the top row of Figure 1 a simulated sample path of the compound Poisson process $J$, with $N\left(0, \frac{1}{2}\right)$ distributed jumps, can be seen over three time scales. The corresponding Lévy process $M$, with parameters $\theta=-0.2$ and $\gamma=0.1$, can be seen in the bottom row. Over all three time intervals we can recognise the desired asymmetry for this set of parameters. If $J$ jumps up then $M$ jumps down and vice versa. If $J$ does not move then we observe the downwards drift of $M$, which can be seen on the right-hand side of Figure 1. 

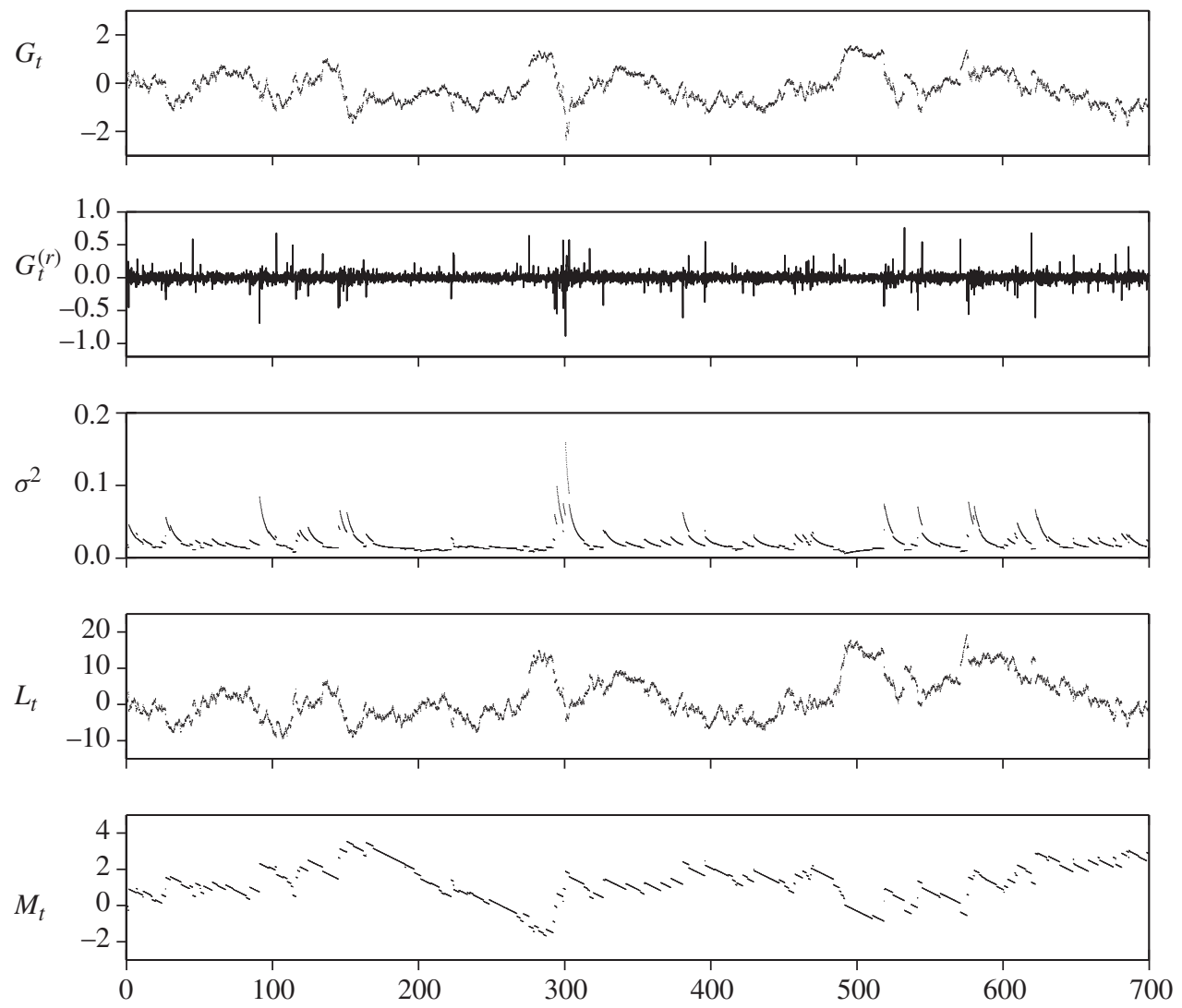

FIGURE 2: Observations of the log-price process $G_{t}$ (top row), the return process $G_{t}^{(r)}$ (second row), the volatility process $\sigma_{t}^{2}$ (third row), with parameters $b_{1}=1, a_{1}=0.1, \mu=-4, \theta=-0.2$, and $\gamma=0.1$, the driving Lévy process $L_{t}$ (fourth row), and the Lévy process $M_{t}$ (bottom row) in the time interval $(0,700]$.

The log-volatility process is then of the form

$$
\begin{aligned}
\log \left(\sigma_{t}^{2}\right)= & \mu+b_{1} \exp \left(-a_{1} t\right) X_{0}+\int_{0}^{t} b_{1} \exp \left(-a_{1}(t-s)\right) \mathrm{d} M_{s} \\
= & \mu+b_{1} \exp \left(-a_{1} t\right) X_{0}+\sum_{k=1}^{N_{t}} b_{1} \exp \left(-a_{1}\left(t-T_{k}\right)\right)\left[\theta Z_{k}+\gamma\left|Z_{k}\right|\right] \\
& -C \frac{b_{1}}{a_{1}}\left(1-\exp \left(-a_{1} t\right)\right) \quad \text { for } t>0
\end{aligned}
$$

and the $\log$-price process is given by

$$
G_{t}=\int_{0}^{t} \sigma_{s-} \mathrm{d} W_{s}+\sum_{k=1}^{N_{t}} \sigma_{T_{k}-} Z_{k}, \quad t>0, G_{0}=0,
$$

with jump times $T_{k}, k \in \mathbb{N}$. 
Generally the simulation of a sample path of the log-price process, $G$, and the log-volatility process, $\log \left(\sigma^{2}\right)$, over a time interval $[0, T]$ is carried out in the following steps.

1. Choose observation times $0=t_{0}<t_{1}<\cdots<t_{n} \leq T$, possibly random.

2. Simulate the jump times $\left(T_{k}\right), k=1, \ldots, n_{T}$, with $n_{T}:=\max \left\{k \in \mathbb{N}: T_{k} \leq T\right\}$, of the compound Poisson process $J$.

3. Approximate the state process (3.4) of the log-volatility by a stochastic Euler scheme.

4. Compute an approximation $\widehat{G}$ via the recursion

$$
\widehat{G}_{t_{i}}=\widehat{G}_{t_{i-1}}+\sqrt{\exp \left(\mu+\boldsymbol{b}^{\top} \widehat{\boldsymbol{X}}_{t_{i-1}}\right)} \widetilde{W}_{i}+\sum_{k=N_{t_{i-1}}+1}^{N_{t_{i}}} \sqrt{\exp \left(\mu+\boldsymbol{b}^{\top} \widehat{\boldsymbol{X}}_{T_{k}-}\right)} Z_{k},
$$

where $\widetilde{W}_{i} \sim N\left(0, t_{i}-t_{i-1}\right)$, and $\widehat{\boldsymbol{X}}_{T_{k}-}$ is the Euler approximation without the jump $\Delta M_{T_{k}}$.

In Figure 2 the results of the above simulation procedure are shown. The jump rate $\lambda$ is now chosen to be $\frac{1}{4}$, which implies a variance of the jump sizes $Z_{i}$ of 4 . For i.i.d. exponentially distributed interarrival times $\Delta t_{i}:=t_{i}-t_{i-1}$, with $\mathrm{E}\left(\Delta t_{i}\right)=1$, the sample path of the logprice $G$, the return process $G^{(\Delta t}$.) , and the volatility process $\sigma^{2}$ are displayed in the first three rows of Figure 2. The sample paths of the Lévy processes $L$ and $M$ are shown in the bottom two rows. From the plots of the return and volatility processes, we see the negative correlation between the two processes. On the one hand, we recognise increases in the volatility after large negative returns, and on the other hand, we recognise a decrease in the volatility after a larger positive return. This displays the leverage effect explained in Subsection 3.1.

\subsection{Mixing}

Mixing properties are useful for a number of applications including asymptotic statistics, since a central limit theorem holds for mixing processes (see [8] for a comprehensive treatment of mixing properties). For an application in this continuous-time GARCH setting, see, e.g. Theorem 3 of Haug et al. [12]. Thus, we will derive mixing properties of the strictly stationary volatility process and the return process in the $\operatorname{ECOGARCH}(p, q)$ model.

First we recall the definition of strong mixing, which is also called $\alpha$-mixing for a process with a continuous-time parameter.

Definition 3.2. ([7].) For a process $Y=\left(Y_{s}\right)_{s \geq 0}$, define the $\sigma$-algebras $\mathcal{F}_{[0, u]}^{Y}:=$ $\sigma\left(\left(Y_{s}\right)_{s \in[0, u]}\right)$ and $\mathcal{F}_{[u+t, \infty)}^{Y}:=\sigma\left(\left(Y_{s}\right)_{s \geq u+t}\right)$ for all $u \geq 0$. Then $Y$ is called strongly or $\alpha$-mixing if

$$
\alpha_{Y}(t):=\sup _{u \geq 0} \alpha\left(\mathcal{F}_{[0, u]}^{Y}, \mathcal{F}_{[u+t, \infty)}^{Y}\right) \rightarrow 0 \quad \text { as } t \rightarrow \infty,
$$

where $\alpha\left(\mathcal{F}_{[0, u]}^{Y}, \mathcal{F}_{[u+t, \infty)}^{Y}\right):=\sup \left\{|\mathrm{P}(A \cap B)-\mathrm{P}(A) \mathrm{P}(B)|: A \in \mathcal{F}_{[0, u]}^{Y}, B \in \mathcal{F}_{[u+t, \infty)}^{Y}\right\}$.

Above we denote by $\sigma(\cdot)$ the generated completed $\sigma$-algebra. The strong mixing property with exponential rate of the log-volatility, volatility, and return processes is the subject of Theorem 3.1, below. Here strong mixing with exponential rate (exponentially $\alpha$-mixing) means that the $\alpha$-mixing coefficient $\alpha$. ( $t$ ) decays to 0 exponentially fast as $t$ tends to $\infty$. 
Theorem 3.1. Let $\log \left(\sigma^{2}\right)$ be as defined by (3.3) and (3.4) with both $\theta$ and $\gamma$ not equal to 0 . Assume that $\mathrm{E}\left(L_{1}^{2}\right)<\infty$, that the eigenvalues of $\boldsymbol{A}$ all have negative real parts, and that $\boldsymbol{X}_{0}$ has the same distribution as $\int_{0}^{\infty} \mathrm{e}^{A u} \mathbf{1}_{q} \mathrm{~d} M_{u}$; hence, $\log \left(\sigma^{2}\right)$ and $\sigma^{2}$ are strictly stationary.

(i) Then there exist constants $K>0$ and $a>0$ such that

$$
\alpha_{\log \left(\sigma^{2}\right)}(t) \leq K \mathrm{e}^{-a t} \quad \text { and } \quad \alpha_{\sigma^{2}}(t) \leq K \mathrm{e}^{-a t} \text { as } t \rightarrow \infty
$$

where $\alpha_{\log \left(\sigma^{2}\right)}(t)$ and $\alpha_{\sigma^{2}}(t)$ are the $\alpha$-mixing coefficients of the log-volatility and volatility processes, respectively.

(ii) Then the discrete-time process $\left(G_{n r}^{(r)}\right)_{n \in \mathbb{N}}$, where $G_{n r}^{(r)}$ is defined in (3.5), is strongly mixing with exponential rate and ergodic.

Proof. (i) The log-volatility process is a $\operatorname{CARMA}(q, p-1)$ process, which is equal to the first component of the $q$-dimensional Ornstein-Uhlenbeck process $V:=\left(V^{1}, \ldots, V^{q}\right)^{\top} \in \mathbb{R}^{q}$ (see, e.g. [4, Section 4]), where, for fixed $t$,

$$
\boldsymbol{V}_{t}=\exp \left(\boldsymbol{B} \boldsymbol{A} \boldsymbol{B}^{-1}(t-s)\right) \boldsymbol{V}_{s}+\int_{s}^{t} \exp (\boldsymbol{A}(t-u)) \boldsymbol{B} \mathbf{1}_{q} \mathrm{~d} M_{u} \quad \text { a.s. }
$$

with

$$
\boldsymbol{B}=\left[\begin{array}{ccccc}
b_{1} & b_{2} & b_{3} & \cdots & b_{q} \\
0 & 1 & 0 & \cdots & 0 \\
0 & 0 & 1 & \cdots & 0 \\
\vdots & \vdots & \vdots & \ddots & \vdots \\
0 & 0 & 0 & \cdots & 1
\end{array}\right]
$$

Since $L$, hence $M$, has finite second moment, $\boldsymbol{V}$ also has finite second moment. Therefore, condition (4.5) of [15] is satisfied. By Theorem 4.3 of [15], $\boldsymbol{V}$ is then exponentially $\alpha$-mixing. Since every component of a multidimensional exponentially strong mixing process is exponentially strong mixing, the log-volatility process is also exponentially $\alpha$-mixing. The property of $\alpha$-mixing is invariant under continuous transformations, which implies that $\sigma^{2}$ also has this property.

(ii) Define the $\sigma$-algebras

$$
\mathcal{F}_{I}^{\sigma^{2}, \mathrm{~d} L}:=\sigma\left(\sigma_{t}^{2}, L_{t}-L_{s}: s, t \in I\right) \text { for } I \subset \mathbb{R}
$$

and

$$
\mathcal{F}_{J}^{G^{(r)}}:=\sigma\left(G_{k r}^{(r)}: k \in J\right) \text { for } J \subset \mathbb{N} .
$$

From (3.5) it follows that

$$
\mathcal{F}_{\{1,2, \ldots, l\}}^{G^{(r)}} \subset \mathcal{F}_{[0, l r]}^{\sigma^{2}, \mathrm{~d} L} \quad \text { and } \quad \mathcal{F}_{\{k+l, k+l+1, \ldots\}}^{G^{(r)}} \subset \mathcal{F}_{[(k+l-1) r, \infty)}^{\sigma^{2}, \mathrm{~d} L} .
$$

To show the strong mixing property of the return process we will use the following relation:

$$
\alpha\left(\mathcal{F}_{1}, \mathcal{F}_{2}\right) \leq \tilde{\alpha}\left(\mathcal{F}_{1}, \mathcal{F}_{2}\right) \leq 6 \alpha\left(\mathcal{F}_{1}, \mathcal{F}_{2}\right),
$$

where $\mathcal{F}_{1}$ and $\mathcal{F}_{2}$ are $\sigma$-algebras,

$$
\tilde{\alpha}\left(\mathcal{F}_{1}, \mathcal{F}_{2}\right):=\sup \left\{\left\|\mathrm{E}\left(f \mid \mathcal{F}_{1}\right)-\mathrm{E}(f)\right\|_{L^{1}(P)}: f \in b \mathcal{F}_{2},\|f\|_{\infty} \leq 1\right\},
$$


$\|\cdot\|_{L^{1}(P)}$ and $\|\cdot\|_{\infty}$ denote the $L^{1}$-norm under $P$ and the sup-norm, respectively, and $b \mathcal{F}$ denotes the set of all bounded $\mathcal{F}$-measurable random variables. The left-hand inequality of (3.9) is easy to see (cf. [12, Lemma B.2]) and the right-hand inequality follows from Lemma 3.5 of [17]. For a stochastic process $Y$, the corresponding $\tilde{\alpha}$-mixing coefficient is defined as

$$
\tilde{\alpha}_{Y}(t):=\sup _{s \in \mathbb{R}_{+}} \tilde{\alpha}\left(\mathcal{F}_{[0, s]}^{Y}, \mathcal{F}_{[s+t, \infty)}^{Y}\right), \quad t \in \mathbb{R}_{+}
$$

see, e.g. [16, Section 2.1]. Now since $\left(G_{n r}^{(r)}\right)_{n \in \mathbb{N}}$ is strictly stationary, we have the following:

$$
\begin{aligned}
\tilde{\alpha}_{G^{(r)}}(k-l) & =\sup \left\{\left\|\mathrm{E}\left(f \mid \mathcal{F}_{\{1,2, \ldots, l\}}^{G^{(r)}}\right)-\mathrm{E}(f)\right\|_{L^{1}(P)}: f \in b \mathcal{F}_{\{k, k+1, \ldots\}}^{G^{(r)}},\|f\|_{\infty} \leq 1\right\} \\
& \leq \sup \left\{\left\|\mathrm{E}\left(f \mid \mathcal{F}_{[0, l r]}^{\sigma^{2}, \mathrm{~d} L}\right)-\mathrm{E}(f)\right\|_{L^{1}(P)}: f \in b \mathcal{F}_{[(k-1) r, \infty)}^{\sigma^{2}, \mathrm{~d} L},\|f\|_{\infty} \leq 1\right\},
\end{aligned}
$$

where the inequality follows from (3.8) and an application of Jensen's inequality (see also [16, Remark 1]). From the exponentially $\alpha$-mixing property of $\sigma^{2}$ and (3.9), we find that there exists a constant $K_{\sigma^{2}}>0$ such that

$$
\tilde{\alpha}_{\sigma^{2}}(t-s)=\sup \left\{\left\|\mathrm{E}\left(f \mid \mathcal{F}_{[0, s]}^{\sigma^{2}}\right)-\mathrm{E}(f)\right\|_{L^{1}(P)}: f \in b \mathcal{F}_{[t, \infty)}^{\sigma^{2}, \mathrm{~d} L},\|f\|_{\infty} \leq 1\right\} \leq K_{\sigma^{2}} \mathrm{e}^{-a(t-s)}
$$

for all $0 \leq s \leq t<\infty$ and $\|f\|_{\infty} \leq 1$. Now analogously to the proof of Lemma 1 of [14], it follows that

$$
\left\|\mathrm{E}\left(f \mid \mathcal{F}_{[0, l r]}^{\sigma^{2}, \mathrm{~d} L}\right)-\mathrm{E}(f)\right\|_{L^{1}(P)} \leq K_{\sigma^{2}} \mathrm{e}^{-a((k-1-l) r)}\|f\|_{\infty}
$$

for all $f \in b \mathcal{F}_{[(k-1) r, \infty)}^{\sigma^{2}, \mathrm{~d} L}$. The only difference is that we do not have a Markov process; hence, we have to condition on the information over the whole time interval $[0, l r]$ and not just on the information at the time point $l r$. This implies that we have

$$
\tilde{\alpha}_{G^{(r)}}(k-l) \leq K_{\sigma^{2}} \mathrm{e}^{-a((k-1-l) r)},
$$

which means that $\left(G_{n r}^{(r)}\right)_{n \in \mathbb{N}}$ is exponentially $\alpha$-mixing by (3.9). Since strict stationarity and strong mixing imply ergodicity the result follows.

\section{Second-order properties of the volatility process}

In this section we derive moments and the autocovariance function of the volatility process $\sigma^{2}$. Since it is a nonlinear transformation of a $\operatorname{CARMA}(q, p-1)$ process, we will first recall the moment structure and conditions for weak stationarity of a $\operatorname{CARMA}(q, p-1)$ process.

Proposition 4.1. If $\boldsymbol{X}_{0}$ has the same mean vector and covariance matrix as $\int_{0}^{\infty} \mathrm{e}^{A u} \mathbf{1}_{q} \mathrm{~d} M_{u}$, then $\log \left(\sigma^{2}\right)$ is weakly stationary. In the weakly stationary case the mean and autocovariance function of $\log \left(\sigma^{2}\right)$ are given by

$$
\mathrm{E}\left(\log \left(\sigma_{t}^{2}\right)\right)=\mu \quad \text { and } \quad \operatorname{cov}\left(\log \left(\sigma_{t+h}^{2}\right), \log \left(\sigma_{t}^{2}\right)\right)=\mathrm{E}\left(M_{1}^{2}\right) \boldsymbol{b}^{\top} \mathrm{e}^{\boldsymbol{A} h} \Sigma \boldsymbol{b}, \quad t, h \geq 0,
$$

where

$$
\Sigma:=\int_{0}^{\infty} \mathrm{e}^{\boldsymbol{A} s} \mathbf{1}_{q} \mathbf{1}_{q}^{\top} \exp \left(\boldsymbol{A}^{\top} s\right) \mathrm{d} s
$$


The results follow from Proposition 1 and Remark 4 of [5] and the fact that $\int_{\mathbb{R}} g(u-h)$ $\times g(u) \mathrm{d} u=\boldsymbol{b}^{\top} \mathrm{e}^{\boldsymbol{A} h} \Sigma \boldsymbol{b}$, with $g$ as defined in (3.7).

The moments of the strictly stationary volatility process are exponential moments of the stationary distribution of the log-volatility process. In Remark 3.2(i) we gave conditions for the existence of a stationary distribution $F$ of the log-volatility process. In Proposition 4.2, below, we want to further characterise this distribution.

Proposition 4.2. Let $\left(\gamma_{M}, 0, v_{M}\right)$ be the characteristic triplet of the Lévy process $M$, where $M$ is as defined in (3.1), and $F$ is the stationary distribution of the log-volatility process. Then $F$ is infinitely divisible with characteristic triplet $\left(\gamma_{\infty}, 0, v_{\infty}\right)$, where

$$
\begin{aligned}
& \gamma_{\infty}=\mu+\int_{0}^{\infty} g(s) \gamma_{M} \mathrm{~d} s+\int_{0}^{\infty} \int_{\mathbb{R}} g(s) x\left[\chi_{(-1,1)}(g(s) x)-\chi_{(-1,1)}(x)\right] v_{M}(\mathrm{~d} x) \mathrm{d} s, \\
& v_{\infty}(B)=\int_{0}^{\infty} \int_{\mathbb{R}} \chi_{B}(g(s) x) v_{M}(\mathrm{~d} x) \mathrm{d} s, \quad B \in \mathscr{B}(\mathbb{R}),
\end{aligned}
$$

Proof. In the strictly stationary case the log-volatility process is the continuous-time moving average process (3.6). Since $M$ has finite variance, the kernel $g$ and the driving Lévy process $M$ satisfy the conditions in Theorem 2.7 of [20] which are:

- $\int_{\mathbb{R}}\left|\gamma_{M} g(s)+\int_{\mathbb{R}} x g(s)\left[\chi_{(-1,1)}(x g(s))-\chi_{(-1,1)}(x)\right] v_{M}(\mathrm{~d} x)\right| \mathrm{d} s<\infty$,

- $\int_{\mathbb{R}} \int_{\mathbb{R}} \min \left(|g(s) x|^{2}, 1\right) v_{M}(\mathrm{~d} x) \mathrm{d} s<\infty$.

Therefore, the stationary distribution $F$ of the log-volatility process is infinitely divisible with characteristic triplet $\left(\gamma_{\infty}, 0, v_{\infty}\right)$.

Let $\log \left(\sigma_{\infty}^{2}\right)$ be a random variable with distribution $F$. Since $F$ is infinitely divisible, we can now apply Theorem 25.17 of [22] to calculate the exponential moments of $\log \left(\sigma_{\infty}^{2}\right)$, i.e. the moments of $\sigma_{\infty}^{2}$, in Proposition 4.3, below.

Proposition 4.3. Let $F$ be the stationary distribution of $\log \left(\sigma^{2}\right)$ with characteristic triplet $\left(\gamma_{\infty}, 0, v_{\infty}\right)$. Then the $k$ th moment of $\sigma_{t}^{2}$ is finite if

$$
\begin{aligned}
k \in K_{\infty} & =\left\{s \in \mathbb{R}: \int_{|x|>1} \mathrm{e}^{s x} v_{\infty}(\mathrm{d} x)<\infty\right\} \\
& =\left\{s \in \mathbb{R}: \int_{0}^{\infty} \int_{x \in \mathbb{R},|h(x)|>1} \mathrm{e}^{s g(u) x} v_{L}(\mathrm{~d} x) \mathrm{d} u<\infty\right\} .
\end{aligned}
$$

In this case

$$
\Psi_{\infty}(k):=\gamma_{\infty} k+\int_{\mathbb{R}}\left(\mathrm{e}^{k x}-1-k x \chi_{(-1,1)}(x)\right) \nu_{\infty}(\mathrm{d} x)
$$

is well defined and

$$
\mathrm{E}\left(\sigma_{t}^{2 k}\right)=\exp \left(\Psi_{\infty}(k)\right) \text { for all } t \geq 0 .
$$

Proposition 4.4. Let $\log \left(\sigma_{t}^{2}\right)$ be the strictly stationary solution of (3.3) and (3.4). Assume that $\mathrm{E}\left(\sigma_{t}^{4}\right)<\infty$ for all $t \geq 0$. Let $\Psi_{\infty}^{h}(\cdot)$ and $\Psi^{h}(\cdot)$ be as defined by (4.1) with kernel function $g$ replaced by $g_{\infty}^{h}(s)=\bar{b}^{\top}\left(I_{q}+\mathrm{e}^{A h}\right) \mathrm{e}^{A s} \mathbf{1}_{q}$ and $g^{h}(s)=\boldsymbol{b}^{\top} \mathrm{e}^{A s} \mathbf{1}_{q} \chi_{(0, h)}(s)$, respectively. Then the autocovariance function of $\sigma^{2}$ is given by the following expression:

$$
\operatorname{cov}\left(\sigma_{t+h}^{2}, \sigma_{t}^{2}\right)=\exp \left(\Psi_{\infty}^{h}(1)\right) \exp \left(\Psi^{h}(1)\right)-\exp \left(2 \Psi_{\infty}(1)\right), \quad h>0, t \geq 0 .
$$


Proof. Let $\widetilde{F}_{t}^{M}=\sigma\left(M_{s},-\infty<s \leq t\right)$ be the $\sigma$-algebra generated by the Lévy process $M$ up to time $t$, then

$$
\begin{aligned}
\mathrm{E}\left(\sigma_{t+h}^{2} \mid \mathcal{F}_{t}^{M}\right)= & \mathrm{E}\left(\exp \left(\mu+\int_{-\infty}^{t+h} g(t+h-s) \mathrm{d} M_{s}\right) \mid \mathcal{F}_{t}^{M}\right) \\
= & \exp \left(\mu+\int_{-\infty}^{t} \boldsymbol{b}^{\top} \mathrm{e}^{A h} \mathrm{e}^{A(t-s)} \mathbf{1}_{q} \mathrm{~d} M_{s}\right) \\
& \times \mathrm{E}\left(\exp \left(\int_{t}^{t+h} g(t+h-s) \mathrm{d} M_{s}\right)\right) .
\end{aligned}
$$

Therefore, we obtain

$$
\begin{aligned}
\mathrm{E}\left(\sigma_{t+h}^{2} \sigma_{t}^{2}\right)= & \mathrm{E}\left(\mathrm{E}\left(\sigma_{t+h}^{2} \sigma_{t}^{2} \mid \mathcal{F}_{t}^{M}\right)\right) \\
= & \mathrm{E}\left(\sigma_{t}^{2} \mathrm{E}\left(\sigma_{t+h}^{2} \mid \mathcal{F}_{t}^{M}\right)\right) \\
= & \mathrm{E}\left(\sigma_{t}^{2} \exp \left(\mu+\int_{-\infty}^{t} \boldsymbol{b}^{\top} \mathrm{e}^{A h} \mathrm{e}^{\boldsymbol{A}(t-s)} \mathbf{1}_{q} \mathrm{~d} M_{s}\right)\right. \\
& \left.\quad \times \mathrm{E}\left(\exp \left(\int_{t}^{t+h} g(t+h-s) \mathrm{d} M_{s}\right)\right)\right) \\
= & \mathrm{E}\left(\exp \left(2 \mu+\int_{-\infty}^{t} \boldsymbol{b}^{\top}\left(I_{q}+\mathrm{e}^{A h}\right) \mathrm{e}^{\boldsymbol{A}(t-s)} \mathbf{1}_{q} \mathrm{~d} M_{s}\right)\right) \mathrm{E}\left(\exp \left(\int_{0}^{h} g(s) \mathrm{d} M_{s}\right)\right) \\
= & \mathrm{E}\left(\exp \left(\mu+\int_{0}^{\infty} \boldsymbol{b}^{\top}\left(I_{q}+\mathrm{e}^{A h}\right) \mathrm{e}^{\boldsymbol{A} s} \mathbf{1}_{q} \mathrm{~d} M_{s}\right)\right) \\
& \times \mathrm{E}\left(\exp \left(\mu+\int_{0}^{\infty} \boldsymbol{b}^{\top} \mathrm{e}^{A s} \mathbf{1}_{q} \chi(0, h)(s) \mathrm{d} M_{s}\right)\right) \\
= & \exp \left(\Psi_{\infty}^{h}(1)\right) \exp \left(\Psi^{h}(1)\right),
\end{aligned}
$$

where the last equality follows from (4.2) when we substitute the kernel $g$ in (3.6) by $g_{\infty}^{h}(s)$ and $g^{h}$, respectively. This together with (4.2) yields (4.3).

\section{Second-order properties of the return process}

In this section we derive the moment structure of the return process

$$
G_{t}^{(r)}=G_{t}-G_{t-r}=\int_{(t-r, t]} \sigma_{s-} \mathrm{d} L_{s}, \quad t \geq r>0 .
$$

We will consider only the case of a strictly stationary volatility process.

\subsection{Moments and autocovariance function of the return process}

Proposition 5.1. Let $L$ be a Lévy process with $\mathrm{E}\left(L_{1}\right)=0$ and $\mathrm{E}\left(L_{1}^{2}\right)<\infty$. Assume that the volatility process $\sigma^{2}$ is strictly stationary with finite mean. Then $\mathrm{E}\left(G_{t}^{2}\right)<\infty$ for all $t \geq 0$ and, for every $t, h \geq r>0$, it holds that

$$
\begin{aligned}
\mathrm{E} G_{t}^{(r)} & =0, \\
\mathrm{E}\left(G_{t}^{(r)}\right)^{2} & =\exp \left(\Psi_{\infty}(1)\right) r \mathrm{E}\left(L_{1}^{2}\right), \\
\operatorname{cov}\left(G_{t}^{(r)}, G_{t+h}^{(r)}\right) & =0 .
\end{aligned}
$$


Furthermore, if $\mathrm{E}\left(L_{1}^{4}\right)<\infty$ and the volatility process has finite second moment then $\mathrm{E}\left(G_{t}^{4}\right)<$ $\infty$ for all $t \geq 0$ and, for every $t, h \geq r>0$, we have

$$
\operatorname{cov}\left(\left(G_{t}^{(r)}\right)^{2},\left(G_{t+h}^{(r)}\right)^{2}\right)=\mathrm{E}\left(L_{1}^{2}\right) \int_{h}^{h+r} \operatorname{cov}\left(G_{r}^{2}, \sigma_{s}^{2}\right) \mathrm{d} s .
$$

Proof. If $L$ has no Brownian component, the proof of (5.1)-(5.3) is analogous to the proof of Proposition 5.1 of [13] and can be extended in the same way as in the proof of Proposition 2.1 of [12] for the case in which $L$ has a Brownian component. Since $G$ is a square integrable martingale, we obtain

$$
\mathrm{E}\left(\left(G_{r}^{(r)}\right)^{2}\left(G_{h+r}^{(r)}\right)^{2}\right)=\mathrm{E}\left(G_{r}^{2}\left(G_{h+r}-G_{h}\right)^{2}\right)=\mathrm{E}\left(G_{r}^{2}\left(G_{h+r}^{2}-G_{h}^{2}\right)\right) .
$$

Using this result,

$$
G_{t}^{2}=2 \int_{0}^{t} G_{s-} \sigma_{s-} \mathrm{d} L_{s}+\int_{0}^{t} \sigma_{s-}^{2} \mathrm{~d}[L, L]_{s}, \quad t \geq 0,
$$

and the compensation formula (see, e.g. [2, Section 0.5]), we obtain

$$
\begin{aligned}
\mathrm{E}\left(\left(G_{r}^{(r)}\right)^{2}\left(G_{h+r}^{(r)}\right)^{2}\right) & =\mathrm{E}\left(2 \int_{h}^{h+r} G_{r}^{2} G_{s-} \sigma_{s-} \mathrm{d} L_{s}+\int_{h}^{h+r} G_{r}^{2} \sigma_{s-}^{2} \mathrm{~d}[L, L]_{s}\right) \\
& =\mathrm{E}\left(\int_{h}^{h+r} G_{r}^{2} \sigma_{s-}^{2} \mathrm{~d}[L, L]_{s}\right) \\
& =\int_{h}^{h+r} \mathrm{E}\left(G_{r}^{2} \sigma_{s}^{2}\right) \tau_{L}^{2} \mathrm{~d} s+\int_{h}^{h+r} \mathrm{E}\left(G_{r}^{2} \sigma_{s}^{2}\right) \mathrm{d} s \int_{\mathbb{R}} x^{2} v_{L}(\mathrm{~d} x) \\
& =\mathrm{E}\left(L_{1}^{2}\right) \int_{h}^{h+r} \mathrm{E}\left(G_{r}^{2} \sigma_{s}^{2}\right) \mathrm{d} s .
\end{aligned}
$$

Hence, the covariance is equal to

$$
\begin{aligned}
\operatorname{cov}\left(\left(G_{r}^{(r)}\right)^{2},\left(G_{h+r}^{(r)}\right)^{2}\right) & =\mathrm{E}\left(\left(G_{r}^{(r)}\right)^{2}\left(G_{h+r}^{(r)}\right)^{2}\right)-\left(\mathrm{E}\left(G_{t}^{(r)}\right)^{2}\right)^{2} \\
& =\mathrm{E}\left(L_{1}^{2}\right) \int_{h}^{h+r}\left(\operatorname{cov}\left(G_{r}^{2}, \sigma_{s}^{2}\right)+\mathrm{E}\left(G_{r}^{2}\right) \mathrm{E}\left(\sigma_{s}^{2}\right)\right) \mathrm{d} s-\left(\mathrm{E}\left(G_{t}^{(r)}\right)^{2}\right)^{2} \\
& =\mathrm{E}\left(L_{1}^{2}\right) \int_{h}^{h+r} \operatorname{cov}\left(G_{r}^{2}, \sigma_{s}^{2}\right) \mathrm{d} s .
\end{aligned}
$$

The covariance is finite if $\mathrm{E}\left(G_{t}^{4}\right)<\infty$ for all $t \geq 0$, and this follows with $\mathrm{E}\left(L_{1}^{4}\right)<\infty$ and $2 \in K_{\infty}$ analogously to the proof of Proposition 1.1 of [12].

Example 5.1. Let us consider again Example 3.1. From 50000 equidistant observations of the simulated log-price process we computed the empirical autocorrelation function of the returns and squared returns. In Figure 3 the first 40 lags of both empirical autocorrelation functions are shown. We recognise the GARCH-like behaviour of zero correlation of the returns and significant correlation of the squared returns. 


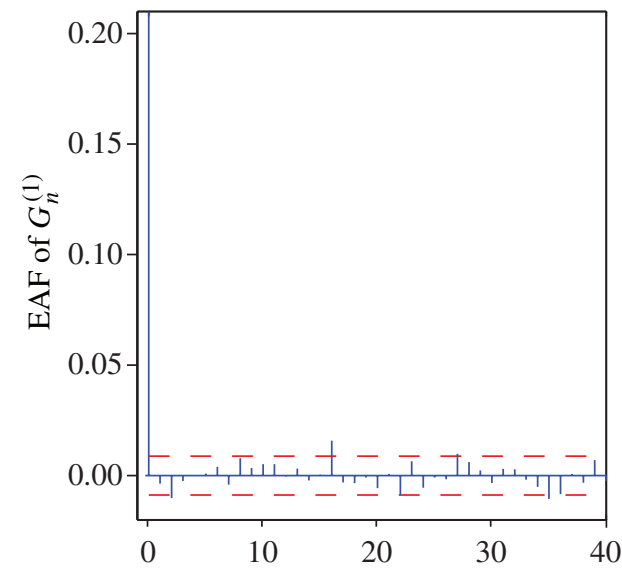

(a)

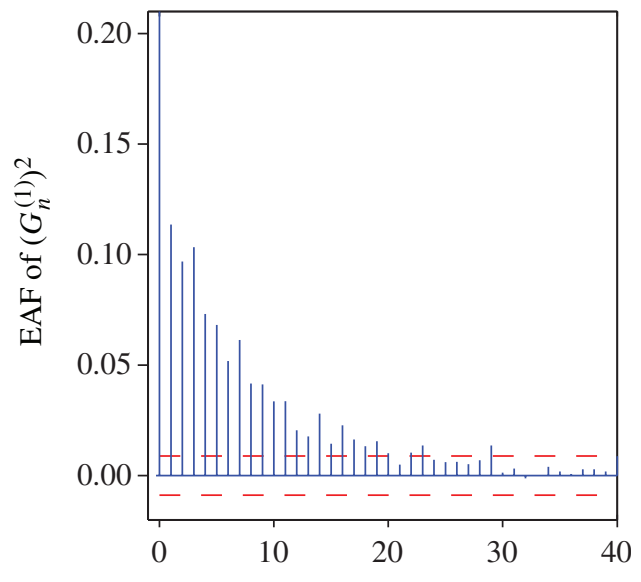

(b)

FIGURE 3: The first 40 lags of the empirical autocorrelation function (EAF) of (a) the return process and (b) the squared return process.

Remark 5.1. In Theorem 3.1 we have seen that the volatility and return processes are strongly mixing with exponential rate. A consequence of this property (see, e.g. [8, Section 1.2.2]) is that there exist constants $K_{1}, K_{2}>0$ such that

$$
\left|\operatorname{cov}\left(\sigma_{t+h}^{2}, \sigma_{t}^{2}\right)\right| \leq K_{1} \mathrm{e}^{-a h} \quad \text { and } \quad\left|\operatorname{cov}\left(\left(G_{(n+h) r}^{(r)}\right)^{2},\left(G_{n r}^{(r)}\right)^{2}\right)\right| \leq K_{2} \mathrm{e}^{-a h}
$$

for all $h>0$, with $a>0$ as given in Theorem 3.1. In particular, this means that the autocovariance function of the volatility and squared returns will decay to 0 at an exponential rate. Therefore, we will speak of a short memory process in both cases. The model can be extended to incorporate long memory effects, by specifing the log-volatility process by a fractionally integrated $\operatorname{CARMA}(q, p-1)$ process. For further details we refer the reader to [11].

\section{Acknowledgements}

We are indebted to two anonymous referees for a number of helpful suggestions and corrections. Furthermore, we would like to thank Claudia Klüppelberg, Alexander Lindner, and Robert Stelzer for fruitful and inspiring discussions. This work was supported by the Deutsche Forschungsgemeinschaft, Sonderforschungsbereich 386, Statistical Analysis of Discrete Structures.

\section{References}

[1] Applebaum, D. (2004). Lévy Processes and Stochastic Calculus. Cambridge University Press.

[2] Bertoin, J. (1996). Lévy Processes. Cambrige University Press.

[3] Bollerslev, T. (1986). Generalized autoregressive conditional heteroskedasticity. J. Econometrics 31, 307-327.

[4] Brockwell, P. J. (2001). Lévy driven CARMA processes. Ann. Inst. Statist. Math. 53, 113-124.

[5] Brockwell, P. J. AND Marquardt, T. (2005). Lévy-driven and fractionally integrated ARMA processes with continuous time parameter. Statist. Sinica 15, 477-494.

[6] Brockwell, P. J., ChadraA, E. and Lindner, A. M. (2006). Continuous time GARCH processes. Ann. Appl. Prob. 16, 790-826.

[7] Davydov, Y. A. (1973). Mixing conditions for Markov chains. Theory Prob. Appl. 18, 312-328. 
[8] Doukhan, P. (1994). Mixing: Properties and Examples (Lecture Notes Statist. 85). Springer, New York.

[9] ENGLE, R. F. (1982). Autoregressive conditional heteroskedasticity with estimates of the variance of UK inflation. Econometrica 50, 987-1008.

[10] Giraitis, L., Leipus, R., Robinson, P. M. and Surgailis, D. (2004). LARCH, leverage, and long memory. J. Financial Econometrics 2, 177-210.

[11] Haug, S. AND CZADO, C. (2006). A fractionally integrated ECOGARCH process. Discussion paper 484, SFB386.

[12] Haug, S., Klüppelberg, C., Lindner, A. M. and Zapp, M. (2007). Method of moment estimation in the COGARCH $(1,1)$ model. Econometrics J. 10, 320-341.

[13] Klüppelberg, C., Lindner, A. And Maller, R. (2004). A continuous time GARCH process driven by a Lévy process: stationarity and second-order behaviour. J. Appl. Prob. 41, 601-622.

[14] Kusuoka, S. AND Yoshida, N. (2000). Malliavin calculus, geometric mixing, and expansion of diffusion functionals. Prob. Theory Relat. Fields 116, 457-484.

[15] Masuda, H. (2004). On multidimensional Ornstein-Uhlenbeck processes driven by a general Lévy process. Bernoulli 10, 97-120.

[16] Masuda, H. (2005). Classical method of moments for partially and discretely observed ergodic models. Statist. Infer. Stoch. Process. 8, 25-50.

[17] MCLeISH, D. L. (1975). Invariance principles for dependent variables. Z. Wahrscheinlichkeitsth. 32, $165-175$.

[18] Nelson, D. B. (1990). ARCH models as diffusion approximations. J. Econometrics 45, 7-38.

[19] Nelson, D. B. (1991). Conditional heteroskedasticity in asset returns: a new approach. Econometrica 59, 347-370.

[20] Rajput, B. S. AND Rosiński, J. (1989). Spectral representations of infinitely divisible processes. Prob. Theory Relat. Fields 82, 451-487.

[21] Robinson, P. M. (1991). Testing for strong serial correlation and dynamic conditional heteroskedasticity in multiple regression. J. Econometrics 47, 67-84.

[22] Sato, K. (1999). Lévy Processes and Infinitely Divisible Distributions. Cambridge University Press.

[23] Surgailis, D. ANd Viano, M. C. (2002). Long memory properties and covariance structure of the EGARCH model. ESAIM Prob. Statist. 6, 311-329. 